\title{
Erratum
}

\section{Solidarity and empowerment in Chicago's Puerto Rican print culture}

Latino Studies (2014) 12, 327. doi:10.1057/lst.2014.35

Correction to: Latino Studies (2014) 12, 88-110. doi: 10.1057/lst.2014.3

In the Acknowledgments section of this article, thanks should have been given to Richard T. Rodríguez. As the result of a typesetting error, his name was incorrectly given as "Richard T. Limön." The publishers apologize for this error. 